\title{
Strong impact of thermal environment on the quantitative genetic basis of a key stress tolerance trait
}

\author{
Michael Ørsted $\mathbb{C}^{1,2} \cdot$ Ary Anthony Hoffmann ${ }^{1,3} \cdot$ Palle Duun Rohde $\mathbb{(}^{4} \cdot$ Peter Sørensen ${ }^{4} \cdot$ \\ Torsten Nygaard Kristensen ${ }^{1,2}$
}

Received: 8 March 2018 / Revised: 20 June 2018 / Accepted: 21 June 2018 / Published online: 26 July 2018

(c) The Genetics Society 2018

\begin{abstract}
Most organisms experience variable and sometimes suboptimal environments in their lifetime. While stressful environmental conditions are normally viewed as a strong selective force, they can also impact directly on the genetic basis of traits such as through environment-dependent gene action. Here, we used the Drosophila melanogaster Genetic Reference Panel to investigate the impact of developmental temperature on variance components and evolutionary potential of cold tolerance. We reared 166 lines at five temperatures and assessed cold tolerance of adult male flies from each line and environment. We show (1) that the expression of genetic variation for cold tolerance is highly dependent on developmental temperature, (2) that the genetic correlation of cold tolerance between environments decreases as developmental temperatures become more distinct, (3) that the correlation between cold tolerance at individual developmental temperatures and plasticity for cold tolerance differs across developmental temperatures, and even switches sign across the thermal developmental gradient, and (4) that evolvability decrease with increasing developmental temperatures. Our results show that the quantitative genetic basis of low temperature tolerance is environment specific. This conclusion is important for the understanding of evolution in variable thermal environments and for designing experiments aimed at pinpointing candidate genes and performing functional analyses of thermal resistance.
\end{abstract}

\section{Introduction}

Stressful environmental conditions not only act as a strong selective force, but also directly affect the genetic

Electronic supplementary material The online version of this article (https://doi.org/10.1038/s41437-018-0117-7) contains supplementary material, which is available to authorised users.

Michael Ørsted

moer@bio.aau.dk

1 Department of Chemistry and Bioscience, Section of Biology and Environmental Science, Aalborg University, Aalborg E 9220, Denmark

2 Department of Bioscience, Section of Genetics, Ecology and Evolution, Aarhus University, Aarhus C 8000, Denmark

3 School of Biosciences, Bio21 Molecular Science and Biotechnology Institute, The University of Melbourne, Parkville, Victoria 3010, Australia

4 Department of Molecular Biology and Genetics, Center for Quantitative Genetics and Genomics, Aarhus University, Tjele 8830, Denmark architecture of traits in several ways. Firstly, stressful conditions can influence the nature of environmental variation, $V_{\mathrm{E}}$ (Hoffmann and Merilä 1999; Ørsted et al. 2018a). Secondly, these conditions can directly affect expression of additive genetic variation, $V_{\mathrm{A}}$ (e.g., Czesak et al. 2006; Wilson et al. 2006). Both changes in $V_{\mathrm{E}}$ and $V_{\mathrm{A}}$ will influence heritability estimates (Gebhardt-Henrich and van Noordwijk 1991; Charmantier and Garant 2005; Wilson et al. 2006; Visscher et al. 2008; Dingemanse et al. 2009; Kristensen et al. 2015). Thirdly, environment-dependent gene action may exist, where the function of genes or gene networks, rather than overall genetic variation, change under stressful conditions (Telonis-Scott et al. 2009). This can lead to a situation where genes influencing a trait in one environment may not have much impact on that trait in a different environment (Sgrò and Hoffmann 2004), which in turn will alter genetic correlations between environments (de Jong 1990; Stearns et al. 1991; Vieira et al. 2000; Stinchcombe et al. 2010). The impact of these environmental effects on genetic variation can be important for evolutionary trajectories (Hoffmann and Merilä 1999; Hercus and Hoffmann 2000), but these mechanisms are 
rarely studied, as they are typically regarded as a nuisance in quantitative genetic models (but see Wilson et al. 2006; Husby et al. 2011; Wood and Brodie 2016).

Environmental conditions are likely to have particularly large effects on the genetic architecture of ecological traits critical for responding to variable environmental conditions. For this class of traits, the environment can influence $V_{\mathrm{A}}, V_{\mathrm{E}}$ and gene action (Sgrò and Hoffmann 2004; Kristensen et al. 2015), affecting the extent to which organisms respond to selection and hence persist in the long run. Tolerance to stressful temperatures in ectotherms, especially in Drosophila, provides examples of traits whose genetic architecture is strongly affected by environmental variation, such as the impact of developmental temperature on the resistance to cold extremes (Schou et al. 2017; Ørsted et al. 2017).

The ways in which the environment influences trait expression across genotypes is often characterised through genotype-by-environment (GxE) interactions, where the direction and magnitude of GxE can depend on genotype (Rohde et al. 2017; Ørsted et al. 2018a) and/or as genetic correlations of a trait measured in several environments (Vieira et al. 2000; Sgrò and Hoffmann 2004; Agrawal and Stinchcombe 2009). Genotypic responses to environmental variation are usually expressed as differences in phenotypic plasticity, which is commonly quantified with linear reaction norms, i.e., the slope of trait values under continuous variation in an environmental variable (Valladares et al. 2006). However, while environmental effects on gene action have typically been investigated by comparing two environments (Telonis-Scott et al. 2009; Ellers and Driessen 2011; Levine et al. 2011; Ketola et al. 2012; Gerken et al. 2015), patterns across environmental gradients are not well established. If the slopes of different genotypes along a gradient vary, there will be GxE interactions and genetic correlations will be less than one. Under a continuous environmental gradient, a polygenic trait might be expected to show declining genetic correlations (increasing GxE interactions) as environments become more dissimilar, assuming that there is an increase in variance due to environment-specific gene expression (Via and Lande 1985; Sgrò and Blows 2004; Spichtig and Kawecki 2004). This could lead to a linear or non-linear decrease in genetic correlations with the environmental variable. Surprisingly, this rather simple prediction has rarely been studied experimentally. Knowledge of the effects of environmental similarity on genetic correlations of stress resistance traits can prove valuable when trying to predict adaptive responses in heterogeneous environments (Sheldon et al. 2003; Sgrò and Blows 2004), such as those arising from anthropogenic climate change (Etterson and Shaw 2001; Agrawal and Stinchcombe 2009).

Environment-dependent gene action also raises the question of how plasticity is linked to stress tolerance in a particular environment. Phenotypic plasticity can be costly, because it requires energy and flexibility of the organism on a number of biological levels (Auld et al. 2010; Tonsor et al. 2013; Murren et al. 2015), and might become maladaptive if environments change rapidly and in unpredictable ways (Kristensen et al. 2008). Several studies have found an inverse association between stress resistance in one environment (here defined as "basal" tolerance) and the capacity for plasticity (Stillman 2003; Nyamukondiwa et al. 2011; Gerken et al. 2015; Comte and Olden 2017; Noh et al. 2017), suggesting a trade-off between the ability to cope with current conditions and performance in an altered environment in the future (Murren et al. 2015). However, other studies have not found evidence for such trade-offs (Kellett et al. 2005; Calosi et al. 2008; Franke et al. 2012; Gunderson et al. 2015; Ørsted et al. 2018a). Thus, it remains unclear if plasticity constrains basal thermal tolerance and vice versa. Adding to the ambiguity, some studies have found that trade-offs are species-specific even across related taxa (Nyamukondiwa et al. 2011; Strachan et al. 2011; Comte and Olden 2017), while others have found seasonal variation in trade-offs (Noh et al. 2017), highlighting the influence of environmental conditions on these patterns.

Here, we investigate the effect of developmental temperature on the genetic architecture of cold tolerance in Drosophila melanogaster. The aims were to explore the environmental dependency of quantitative genetic parameters of cold tolerance, and potential trade-offs between cold tolerance in each environment and plasticity in this trait. We used the Drosophila melanogaster Genetic Reference Panel (DGRP; Mackay et al. 2012; Huang et al. 2014), which was established from a natural population of D. melanogaster from Raleigh, USA. The DGRP consists of a collection of lines that has been inbred to an expected inbreeding coefficient of $F \sim 1$, resulting in extremely high homozygosity throughout the genome within each line. Thus, by assessing cold tolerance of the same DGRP genotype reared in different thermal environments, knowledge about the genetic aspects of cold tolerance, and the association between cold tolerance at individual temperatures and plasticity, can be obtained. Low temperatures limit the geographical distribution of many species more than warm temperatures (Sunday et al. 2011; Williams et al. 2014), and cold tolerance is therefore a good predictor of present and future distributions of species (Kimura 2004; Overgaard et al. 2011; Araújo et al. 2013). The temperature regimes employed in this study were well within the range of what the cosmopolitan D. melanogaster will experience in its natural habitats (Hoffmann et al. 2003), thus providing ecological relevance to our experimental setup. However, it can be difficult to ascertain which of the thermal environments are optimal and which imposes a level of stress, as this is highly dependent on the biological context and traits investigated (David et al. 1997; Hoffmann 2010). 
We reared 166 DGRP lines at five different developmental temperatures and quantified cold tolerance in adults from each developmental temperature. We performed quantitative genetic analyses to estimate genetic and environmental variance components, heritabilities, evolvabilities and phenotypic/genetic correlations of cold tolerance in each developmental environment and for (slope-based) plasticity. This allowed three main questions to be answered. (1) To what extent is cold tolerance phenotypically and genetically correlated between developmental environments, and are these correlations dependent on environmental similarity? (2) Do the developmental temperatures impact on variance components, heritability and evolvability of cold tolerance within developmental temperatures, and on plastic cold tolerance? (3) Is there a trade-off between cold tolerance at individual developmental temperatures and the plasticity of cold tolerance, and is this trade-off dependent on the environment?

\section{Materials and methods}

\section{Experimental setup}

This study included 166 lines from the D. melanogaster Genetic Reference Panel (DGRP) obtained from Bloomington Drosophila Stock Centre (NIH P40OD018537), maintained at $23{ }^{\circ} \mathrm{C}, 50 \%$ relative humidity and a $12: 12 \mathrm{~h}$ photoperiod on a standard oatmeal-sugar-agar-yeast medium for two generations before starting the experiment. The experimental procedure follows the setup previously described in Ørsted et al. (2018a). In summary, we reared each DGRP line at five different temperatures: 17, 20, 23, 26 and $29^{\circ} \mathrm{C}$, from eggs to adults in vials containing $7 \mathrm{~mL}$ standard medium (for details on medium composition see Ørsted et al. 2018b). Eggs were laid by approximately 20 adult flies in three $12 \mathrm{~h}$ periods. Vials were checked daily and eclosed flies were sexed under $\mathrm{CO}_{2}$ anaesthesia. Males were transferred to vials containing fresh medium, and kept at the respective developmental temperature for $48 \mathrm{~h}$. At age $60 \pm 12 \mathrm{~h}$, the cold tolerance of around 10 males (for exact numbers see Table S1) per DGRP line per temperature were assessed using the dynamic measure Critical Thermal Minimum ( $\mathrm{CT}_{\text {min }}$; Overgaard et al. 2012). In this standardised procedure, individual flies were placed in glass vials in a water bath pre-set to $23^{\circ} \mathrm{C}$. Water in the bath was gradually cooled at a rate of $0.1^{\circ} \mathrm{C} / \mathrm{min}$. The temperature at which a fly enters chill coma, i.e., when all movement ceases, was recorded and defines $\mathrm{CT}_{\min }$. In total, 7690 flies were scored for $\mathrm{CT}_{\min }$ by the same person to reduce potential scoring bias. We chose males because virginity is not likely to impact on males to the same extent as it does to females (Goenaga et al. 2012), and we were not able to assure that all flies were virgins when assessed.

Phenotypic plasticity of cold tolerance was estimated as the regression coefficient of a linear regression, i.e., slope of $\mathrm{CT}_{\text {min }}$ on rearing temperature. Linear regression analysis provided the best fit compared to polynomial regression, as assessed by the coefficient of determination (results not shown). In addition, linear norms-of-reaction are the most commonly used in studies of phenotypic plasticity (Valladares et al. 2006), and especially for the relationship between $\mathrm{CT}_{\text {min }}$ and developmental temperature, which is typically linear in Drosophila (Schou et al. 2017; Ørsted et al. 2018a).

\section{Quantitative genetic analyses}

Genetic analyses was performed with the average information restricted maximum-likelihood (AI-REML) procedure (Madsen et al. 1994) utilising the R package QGG (http://psoerensen.github.io/qgg/) that links to DMU (http:// dmu.agrsci.dk/DMU/). We estimated variance components, genetic correlations and broad and narrow sense heritabilities ( $H^{2}$ and $h^{2}$, respectively) based on multi-trait linear mixed models. For $\mathrm{CT}_{\text {min }}$ we fitted a five-trait multi-trait model including all developmental temperatures, and to estimate genetic correlations between $\mathrm{CT}_{\text {min }}$ at individual temperatures and plasticity we fitted a bivariate model. The multi-trait model including $i$-traits was generalised as:

$$
\left[\begin{array}{c}
\mathbf{y}_{1} \\
\vdots \\
\mathbf{y}_{i}
\end{array}\right]=\left[\begin{array}{cc}
\mathbf{X}_{1} & \mathbf{b}_{1} \\
\vdots & \vdots \\
\mathbf{X}_{i} & \mathbf{b}_{i}
\end{array}\right]+\left[\begin{array}{cc}
\mathbf{Z}_{1} & \mathbf{g}_{1} \\
\vdots & \vdots \\
\mathbf{Z}_{i} & \mathbf{g}_{i}
\end{array}\right]+\left[\begin{array}{c}
\mathbf{e}_{1} \\
\vdots \\
\mathbf{e}_{i}
\end{array}\right]
$$

where, $\mathbf{y}_{i}$ was a vector of phenotypic observations (i.e., either temperature specific $\mathrm{CT}_{\text {min }}$ or plasticity), $\mathbf{X}_{i}$ and $\mathbf{Z}_{i}$ are design matrices linking fixed and random genetic effects to the phenotypes. The $\mathbf{b}_{i}$ is a vector of fixed effects (experimental day, water bath, time on day (for $\mathrm{CT}_{\min }$ ), Wolbachia infection status, and five major chromosomal inversions (only when estimating the additive genetic variance), and $\boldsymbol{e}_{i}$ is a vector of random residuals $\left(\boldsymbol{e}_{i} \sim \boldsymbol{N}\left(\boldsymbol{0}, \boldsymbol{I} \sigma_{\boldsymbol{e}_{i}}^{2}\right)\right)$. Estimates of the genetic variance components (and therefore $H^{2}$ ) were obtained by assuming the DGRP lines to be independent, which we modelled by an identity block matrix $\left(\boldsymbol{I}_{L}\right)$ as (co)variance structure of the genetic values, $g \sim N\left(0, \boldsymbol{I}_{L} \sigma_{g}^{2}\right)$. To estimate the additive genetic variance components (including $h^{2}$ ) we estimated the realised relationship among DGRP lines from publicly available single nucleotide polymorphisms (SNPs). Thus $h^{2}$ was the proportion of phenotypic variance captured by common segregating SNPs within the DGRP. We used 


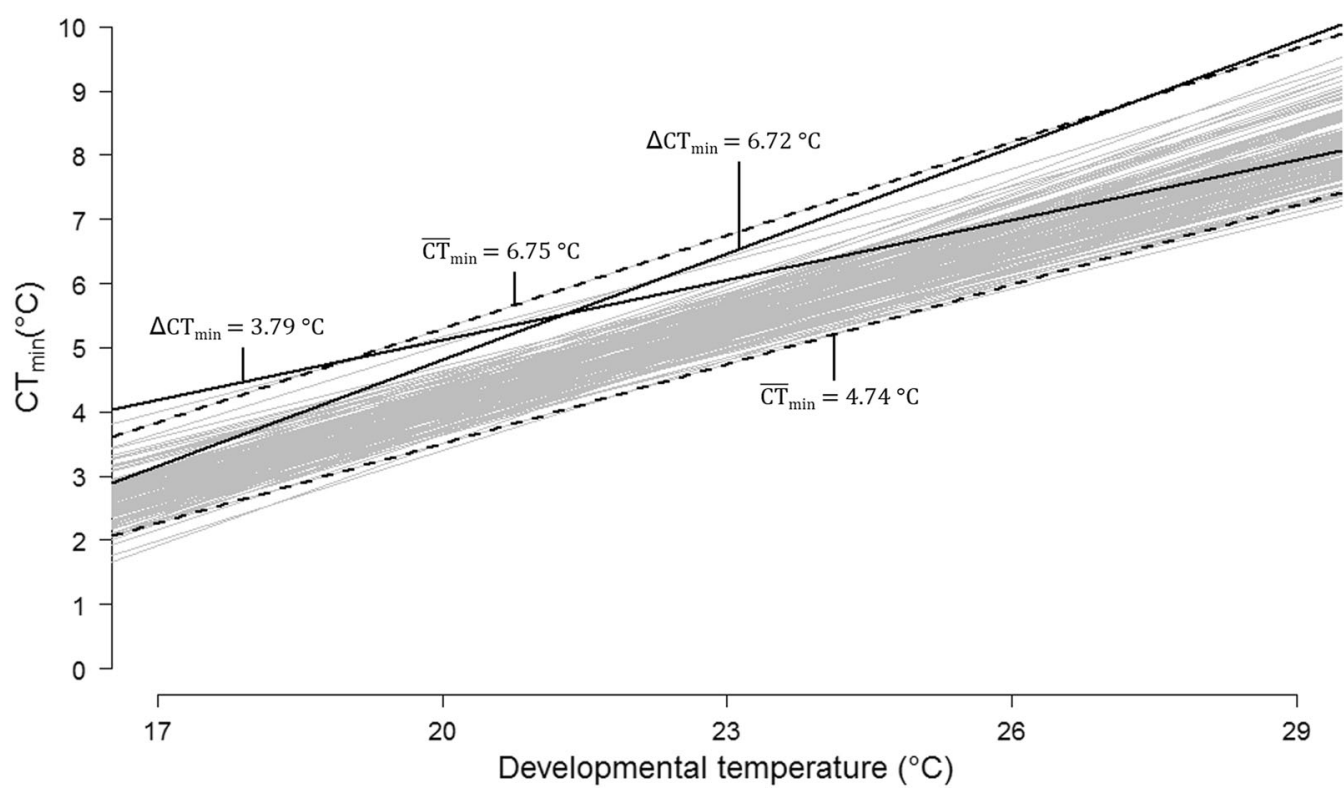

Fig. 1 Linear regressions of critical thermal minimum $\left(\mathrm{CT}_{\min }\right)$ across the five developmental temperatures. Each grey line represents one DGRP line $(n=166)$. The two full black lines represent the DGRP line with the steepest slope and the DGRP line with the flattest slope, with the difference in $\mathrm{CT}_{\min }$ from developing at $17-29^{\circ} \mathrm{C}$ indicated as
$\Delta \mathrm{CT}_{\min }$. The two dashed black lines represent the DGRP lines with the highest and lowest average $\mathrm{CT}_{\min }$ across the temperatures indicated as $\overline{\mathrm{CT}}_{\min }$. These latter two DGRP lines represent a genotype that performs poorly across environments and a genotype that performs well across environments standard filtering for the DGRP, i.e., only considering biallelic SNPs with minor allele frequencies $\geq 0.05$, and for which the Phred scaled variant quality was greater than 500 , and the genotype call $\geq 0.8$ (Mackay et al. 2012; Huang et al. 2014). This yielded $1,725,755$ genetic markers distributed on the six chromosome arms (2L, 2R, 3L, 3R, 4 and $\mathrm{X})$. The notation is similar as above, however, here we included five major polymorphic inversions present in the DGRP system; I2Lt, I2Rns, I3Rp, I3Rk and I3RMo), $\boldsymbol{g}_{i}$ was a vector of random genetic effects, $\boldsymbol{g}_{i} \sim N\left(0, \boldsymbol{G} \sigma_{g_{i}}^{2}\right)$, where $\boldsymbol{G}$ was the additive genomic relationship matrix, and $\boldsymbol{e}_{i}$ was a random residual, $\boldsymbol{e}_{i} \sim N\left(0, \boldsymbol{I} \sigma_{e_{i}}^{2}\right)$. The $\boldsymbol{G}$ matrix was computed as $\boldsymbol{G}=\boldsymbol{W} \boldsymbol{W}^{\prime} / m$ (VanRaden 2008), where $m$ was the number of genetic markers, and $\boldsymbol{W}$ was a centred and scaled genotype matrix. Each column vector of $\boldsymbol{W}, \boldsymbol{w}_{i}=\frac{\boldsymbol{a}_{i}-2 p_{i}}{\sqrt{2 p_{i}\left(1-p_{i}\right)}}, p i$ was the frequency of the minor allele at locus $i$, and $\boldsymbol{a}_{i}$ was the $i$ th column vector of the allele count matrix, $\boldsymbol{A}$, with the genotypes encoded as 0,2 , referring to the number of the minor allele. The SNP data, including karyotype inversions and Wolbachia infection status, can be obtained from the DGRP2 website (http://dgrp2.gnets.ncsu.edu). To achieve a replicated structure of the plasticity measures, which was needed to obtain accurate estimates of the genetic parameters, we grouped the $\mathrm{CT}_{\text {min }}$ data within each DGRP line into three groups of individuals according to day of assay, time of day, and water bath. Based on these replicate groups, we obtained three replicates measures of slope per DGRP line. The slope estimates were robust against resampling of random individuals into groups of the same size (for details see Ørsted et al. 2018a).

Phenotypic correlations were computed as Pearson's correlation using within DGRP line means, and standard errors were estimated as: $\mathrm{SE}_{r}=\sqrt{\frac{1-r^{2}}{n-2}}$, where $n$ was the sample size. We also computed correlations between the rank order of the DGRP lines $\left(r_{r}\right)$, in which lines are ranked within rearing regimes from 1 to 166 .

All variance components were tested under the null hypothesis of being zero using Wald's test. In addition, we tested if correlations differed from zero, i.e., the estimate deviated more than $1.645 \times$ SE from $0(p<0.05)$. We performed pairwise comparisons of genetic correlations, $r_{g}$ among $\mathrm{CT}_{\min }$ in the five rearing temperatures (e.g., $r_{g}(17$, $\left.20^{\circ} \mathrm{C}\right)$ vs. $r_{g}\left(17,23^{\circ} \mathrm{C}\right)$ ), as well as between correlations between $\mathrm{CT}_{\min }$ in each environment and plasticity across environments. These comparisons were based on Fisher's $z$ transformation. Evolvability $\left(I_{\mathrm{A}}\right)$ was computed at each developmental temperature as: $I_{\mathrm{A}}=\widehat{V}_{\mathrm{A}} /\left(\overline{\mathrm{CT}}_{\text {min }}\right)^{2} \times 100$, where $\overline{\mathrm{CT}}_{\text {min }}$ is the mean $\mathrm{CT}_{\text {min }}$ across all DGRP lines in a given environment (Houle 1992; Hoffmann et al. 2016). All analyses were done within the $\mathrm{R}$ programming environment (R Core Team 2017). 
Table 1 Mean $\pm \mathrm{SE}$, minimum, and maximum $\mathrm{CT}_{\min }$ at the five developmental temperatures (17, $20,23,26$ and $29^{\circ} \mathrm{C}$ ) and slope of $\mathrm{CT}_{\text {min }}$ across the thermal environments for all DGRP lines $(n=166)$

\section{Results}

\section{Heritable variation for cold tolerance at the individual rearing temperatures and plasticity}

We observed a significant increase in line mean $\mathrm{CT}_{\text {min }}$, i.e., a decrease in cold tolerance, with increasing rearing temperature (Fig. 1, Table $1, F_{4,804}=3956 ; p<0.001$ ). Average $\mathrm{CT}_{\min }( \pm \mathrm{SE})$ across DGRP lines spanned from $2.81 \pm$ $0.03{ }^{\circ} \mathrm{C}$ for flies reared at 17 to $7.80 \pm 0.04{ }^{\circ} \mathrm{C}$ for flies reared at $29^{\circ} \mathrm{C}$, with considerable variation among DGRP lines within rearing environment (Fig. 2, Table 1 and Table $\mathrm{S} 1)$. The estimates of $H^{2}$ and $h^{2}$ for $\mathrm{CT}_{\min }$ were consistent across the developmental temperatures, with estimates in the range of $0.41-0.48$ and $0.27-0.34$ for $H^{2}$ and $h^{2}$, respectively (Table 2). Plasticity of cold tolerance was estimated as the slope of a linear regression of $\mathrm{CT}_{\min }$ as a function of developmental temperature. The slope was significantly different from zero in all DGRP lines $\left(t_{(3-21)}>\right.$ $2.13 ; p<0.001)$. A significant interaction between line and developmental temperature (two-way ANOVA; $F_{(165,477)}=$ 2.22, $p<0.001)$ indicated a GxE interaction, reflecting differences among DGRP lines in slope ranging from 0.32 to $0.57{ }^{\circ} \mathrm{C}$ in $\mathrm{CT}_{\min }$ per $1{ }^{\circ} \mathrm{C}$ increase in developmental temperature. The heritability estimates for plasticity of $\mathrm{CT}_{\text {min }}$ were 0.65 and 0.51 for $H^{2}$ and $h^{2}$, respectively. We observed a higher total genetic variance $\left(\widehat{V}_{\mathrm{G}}\right)$, additive genetic variance $\left(\widehat{V}_{\mathrm{A}}\right)$ and environmental variance $\left(\widehat{V}_{\mathrm{E}}\right)$ of $\mathrm{CT}_{\text {min }}$ at $29^{\circ} \mathrm{C}$ than at $26^{\circ} \mathrm{C}$ (Table 2). At 17,20 and $23^{\circ} \mathrm{C}$, we observed a decrease in all variance components as compared to $29^{\circ} \mathrm{C}$ (Table 2). In contrast, for evolvability $\left(I_{\mathrm{A}}\right.$; mean corrected $\left.\widehat{V}_{\mathrm{A}}\right)$ of $\mathrm{CT}_{\text {min }}$, we observed higher values at lower temperatures, i.e., from 0.67 at 17 to 0.18 at $29^{\circ} \mathrm{C}$ (Table 2).

\section{Correlations between $\mathrm{CT}_{\min }$ from different developmental temperatures increased with environmental similarity}

We found strong positive genetic correlations $\left(r_{g}\right)$ between $\mathrm{CT}_{\text {min }}$ for DGRP lines at the different rearing temperatures (Fig. 2). The $r_{g}$ values were inversely proportional to the difference between developmental temperatures, i.e., correlations between similar developmental temperatures were larger than between dissimilar developmental temperatures. This pattern was confirmed by statistical pairwise comparisons of $r_{g}$. The phenotypic correlations $\left(r_{p}\right)$ followed the same pattern, decreasing with increasing environmental dissimilarity (Fig. 2). Similarly, the rank order of the DGRP lines was more positively correlated across thermal environments that were more similar to each other (Fig. 2).

\section{Trade-offs between $\mathrm{CT}_{\min }$ at individual developmental temperatures and plastic cold tolerance depended on the environment}

The phenotypic $\left(r_{p}\right)$ correlations between $\mathrm{CT}_{\min }$ at the individual temperatures and slope of $\mathrm{CT}_{\text {min }}$ across temperatures depended on the environment. At $17^{\circ} \mathrm{C}$, the correlation was significantly negative (Fig. 3), i.e., in this environment genotypes with a high cold tolerance (low $\mathrm{CT}_{\text {min }}$ ) tended to have a high plasticity of cold tolerance. However, we did not find this association at $20^{\circ} \mathrm{C}$. At higher temperatures, correlations were significantly positive (Fig. 3), thus genotypes with a high cold tolerance (low $\mathrm{CT}_{\text {min }}$ ) tended to have low plasticity of cold tolerance, suggesting a trade-off between inherent cold tolerance at these developmental temperatures and plasticity. The genetic correlations $\left(r_{g}\right)$ followed the same pattern, particularly when involving $\mathrm{CT}_{\min }$ scores following development at 17,26 and $29^{\circ} \mathrm{C}$ (Fig. 3).

\section{Discussion}

In the present study, we investigated the effects of developmental temperature on quantitative genetic parameters of cold tolerance, which is a key stress tolerance trait. We showed that environmental conditions can directly influence the quantitative genetic basis of cold tolerance by affecting variance components, especially $\widehat{V}_{\mathrm{A}}$. It is expected that if standing genetic variation is environmentally dependent, selection responses will also be affected (Roff 2002; Bijlsma and Loeschcke 2005; Wilson et al. 2006; Husby et al. 2011; Wood and Brodie 2016). This has been demonstrated in recent studies showing that genetic variation and evolutionary potential of stress resistance, morphological traits and life-history traits depend on the environmental conditions (van Heerwaarden and Sgrò 2011; Bubliy et al. 2012; Kristensen et al. 2015; Bastide et al. 2016; Zhao et al. 2016; Feldman et al. 2017). Such 

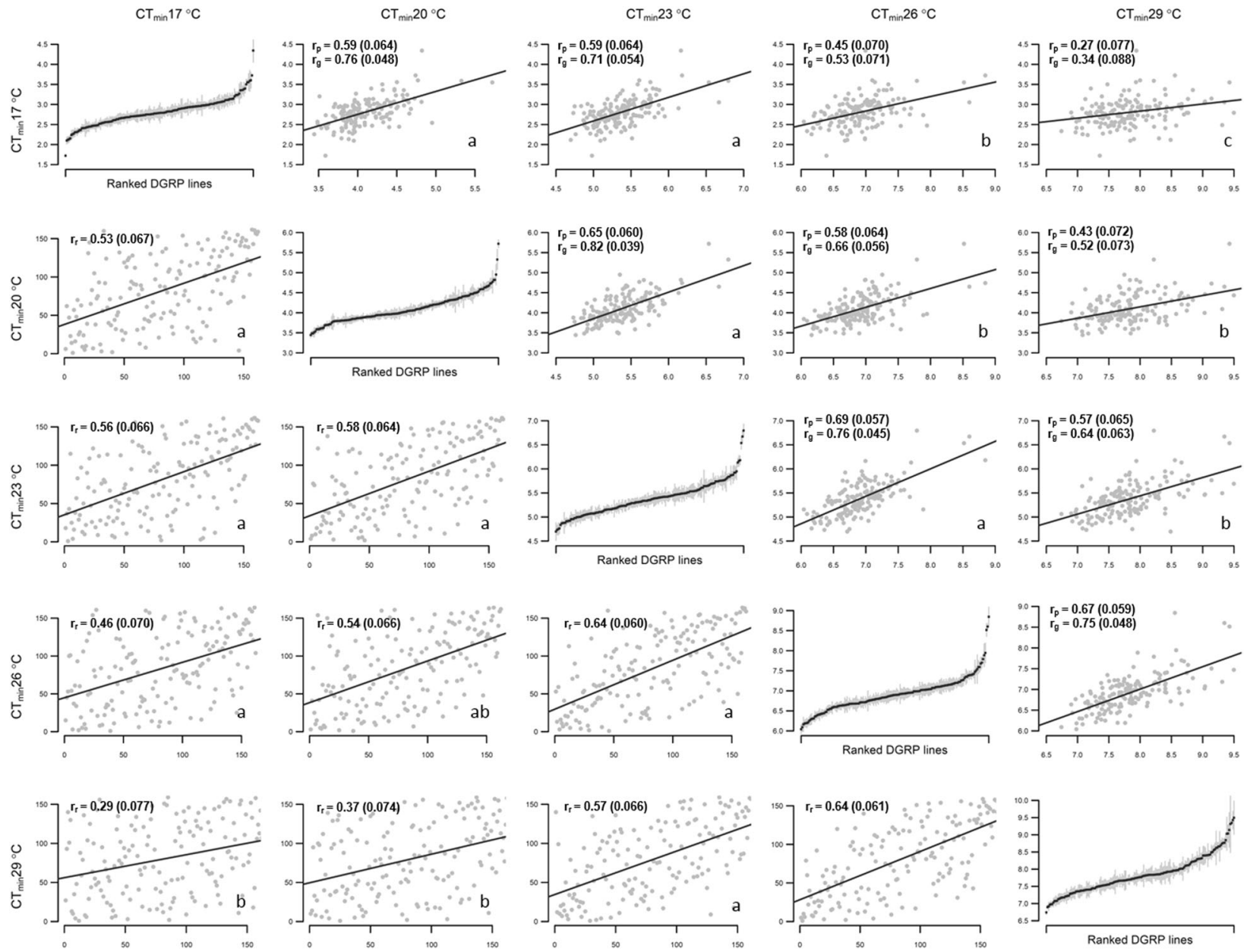

Fig. 2 Correlations of $\mathrm{CT}_{\text {min }}$ across developmental thermal environments. Diagonal elements show the DGRP line mean $\mathrm{CT}_{\min }( \pm \mathrm{SE})$ sorted by increasing values within rearing regime. Plots above the diagonal show the phenotypic correlations, $r_{p}$ (SE in parenthesis) along with the printed genetic correlations, $r_{g}$ (SE in parenthesis) between $\mathrm{CT}_{\min }$ for pairs of environments. Plots below the diagonal show the correlations between the rank order of DGRP lines, $r_{r}$ (SE in parenthesis). In these plots, the DGRP lines are ranked within rearing regimes from 1 to 166. All correlations are Pearson's product moment correlations. In all plots above and below the diagonal, the grey dots represent one DGRP line. Black lines are the regression lines

findings can be explained by environment-specific gene expression, i.e., the impact of genes on a trait can depend on prior environmental exposures. This highlights the need to perform experiments and field studies under environmental conditions that are relevant to organisms at the time traits are under selection (Hoffmann and Parsons 1991; Hoffmann and Merilä 1999). This is important not only from an ecological and evolutionary perspective (Bay et al. 2017) but also for animal and plant breeding, in which effects of the environment on the genetic basis of production traits pose a challenge, such as for genomic selection in heterogeneous environments (Kadarmideen et al. 2006; Zhao et al. 2016). visualising the correlations. All correlations are significantly different from zero, i.e., the estimate deviate more than $1.645 \times \mathrm{SE}$ from 0 $(p<0.05)$. In the lower right corners of the above diagonal plots, the genetic correlations, $r_{g}$ are compared pairwise within rows, and significantly different correlations are denoted with separate letters, e.g., $r_{g}\left(17,20^{\circ} \mathrm{C}\right)$ vs. $r_{g}\left(17,23^{\circ} \mathrm{C}\right)$. In the below diagonal plots, the letters represent significant differences in pairwise comparisons of rank correlations, $r_{r}$ within columns. Some correlations have more letters as they are similar to some but different from other correlations. Comparisons of these correlations are based on Fisher's $z$ transformation $(n=166)$

It has long been debated whether some types of environmental conditions, and particularly stressful or benign conditions, affect heritable variation in distinct ways (Hoffmann and Parsons 1991; Møller and Swaddle 1998). Here we showed an increase in $\widehat{V}_{\mathrm{A}}$ of cold resistance with increasing developmental temperature, and heritability estimates being similar across developmental temperatures (Table 2). However, for mean corrected $\widehat{V}_{\mathrm{A}}$, (i.e., evolvability $\left(I_{\mathrm{A}}\right)$ ), we found higher values at 17 and $20^{\circ} \mathrm{C}$ than in the range $23-29^{\circ} \mathrm{C}$, suggesting that the evolutionary capacity for increasing cold tolerance might be more restricted in warm than in cold environments. This is supported by recent Drosophila studies showing that the 
Table 2 Estimated variance components (genetic variance, $\widehat{V}_{\mathrm{G}}$, environmental variance, $\widehat{V}_{\mathrm{E}}$, and additive genetic variance, $\widehat{V}_{\mathrm{A}}$ ) and broad and narrow sense heritability estimates $\left(H^{2}\right.$ and $h^{2}$, respectively) \pm standard errors (SE), as well as evolvability $\left(I_{\mathrm{A}}=\widehat{V}_{\mathrm{A}} /\left(\operatorname{mean} \mathrm{CT}_{\text {min }}\right)^{2} \times 100\right)$ for CT at the five developmental temperatures and for the slope of $\mathrm{CT}_{\min }$ across temperatures

\begin{tabular}{lllllll}
\hline Trait & $\widehat{V}_{\mathrm{G}}$ & $\widehat{V}_{\mathrm{E}}$ & $\widehat{V}_{\mathrm{A}}$ & $H^{2}$ & $h^{2}$ \\
\hline $\mathrm{CT}_{\min } 17^{\circ} \mathrm{C}$ & $0.1005 \pm 0.00101$ & $0.1437 \pm 0.00042$ & $0.0528 \pm 0.00055$ & $0.41 \pm 0.005$ & $0.27 \pm 0.004$ & 0.669 \\
$\mathrm{CT}_{\min } 20^{\circ} \mathrm{C}$ & $0.0973 \pm 0.00094$ & $0.1078 \pm 0.00031$ & $0.0505 \pm 0.00051$ & $0.47 \pm 0.005$ & $0.32 \pm 0.004$ & 0.302 \\
$\mathrm{CT}_{\min } 23^{\circ} \mathrm{C}$ & $0.0929 \pm 0.00091$ & $0.1131 \pm 0.00033$ & $0.0503 \pm 0.00051$ & $0.45 \pm 0.005$ & $0.31 \pm 0.004$ & 0.173 \\
$\mathrm{CT}_{\min } 26^{\circ} \mathrm{C}$ & $0.1485 \pm 0.00142$ & $0.1581 \pm 0.00046$ & $0.0821 \pm 0.00082$ & $0.48 \pm 0.005$ & $0.34 \pm 0.004$ & 0.172 \\
$\mathrm{CT}_{\min } 29^{\circ} \mathrm{C}$ & $0.1943 \pm 0.00198$ & $0.2703 \pm 0.00083$ & $0.1116 \pm 0.00118$ & $0.42 \pm 0.006$ & $0.29 \pm 0.005$ & 0.184 \\
Slope & $0.0017 \pm 0.00001$ & $0.0009 \pm 0.000005$ & $0.0009 \pm 0.00001$ & $0.65 \pm 0.036$ & $0.51 \pm 0.041$ & 0.519 \\
\hline
\end{tabular}

All variance components were significantly different from zero (Wald's test).

potential for evolution is lower in relatively warmer conditions (Schou et al. 2014; Kristensen et al. 2015). In nature, individuals reared under cold conditions are more likely to encounter subsequent extreme cold conditions, therefore stronger selection for cold tolerance might be expected in natural populations experiencing low developmental temperatures compared to in hotter environments (Ayrinhac et al. 2004). Thus, in the case of cold tolerance, developmental environments with low temperatures might be considered more optimal than those with high temperatures. However, because we did not have a direct measure of stress in each thermal environment, we cannot confidently determine whether the patterns in $\widehat{V}_{\mathrm{A}}$ and $I_{\mathrm{A}}$ are associated with unfavourable environmental conditions (Hoffmann and Merilä 1999; Swindell and Bouzat 2006; Talloen et al. 2009).

The environment can also affect genetic variation via environment-dependent gene action, where genes influencing a certain trait in one environment may not be important for that trait in a different environment. In such cases selection responses can be slowed or even become maladaptive (Steinger et al. 2003). This will often be expressed as altered genetic correlations either between different traits in one environment or between the same trait in a range of environments (Vieira et al. 2000; Sgrò and Hoffmann 2004; Agrawal and Stinchcombe 2009). Here we found positive genetic and phenotypic correlations between cold tolerance across environments, i.e., genotypes with high cold tolerance when developed at one temperature also tended to have high cold tolerance when developed at other temperatures, implying an overlap in the sets of alleles influencing cold tolerance across developmental thermal environments (Via and Lande 1985; Falconer and Mackay 1996; Agrawal and Stinchcombe 2009). However, we also showed that the magnitude of correlations between thermal environments decreased as developmental temperatures diverged. This suggests a shared genetic basis for cold tolerance between rearing environments, which contributes less to genetic variation as environments become more dissimilar. Although evidence for such patterns has rarely been investigated, a few studies have found similar results; Sgrò and Blows (2004) found declining genetic correlations with environmental dissimilarity in three life-history traits in Drosophila serrata, while Stinchcombe et al. (2010) found declining genetic correlations with environmental distance for growth rate in jewelweed.

Our findings are in line with other studies suggesting that the genetic architecture of fitness components is specific to an environment (Bourret and Garant 2015; Rellstab et al. 2017). This represents a challenge when testing for adaptation at specific loci which often seem to be environment and population specific (Zhao et al. 2011; Manenti et al. 2016). Knowledge about the genetic architecture of many complex traits has been rapidly accumulating recently, aided by developments in genomics and quantitative genetics. Typically, such studies identify genes, variance components and heritabilities for a given trait in one environment to predict evolutionary trajectories. In animal and plant breeding, such knowledge may be used to predict selection responses and devise genomic selection procedures. However, our results show that the architecture of an important stress resistance trait is highly dependent on environmental conditions, challenging the notion that there are candidate genes and a particular architecture applicable across a gradient of environmental conditions.

An important issue for the evolution of thermal resistance is whether an organism's thermotolerance is constrained by plasticity, particularly in response to anthropogenic climate change (Stillman 2003; Chown et al. 2010; Levine et al. 2011; Gunderson et al. 2015; Comte and Olden 2017). Some studies point to a negative association between an organism's thermal tolerance and plasticity in tolerance (Hoffmann et al. 2003; Kellett et al. 2005; Nyamukondiwa et al. 2011; Gerken et al. 2015; Noh et al. 2017). Here we showed that any association between cold tolerance and plasticity depend on the environment; both the phenotypic and genetic correlations shifted in sign as developmental temperatures shifted from 17 to $29^{\circ} \mathrm{C}$ (Fig. 3). Given that the lowest $\mathrm{CT}_{\text {min }}$ occurred at the lowest developmental temperature, genotypes with a flat slope 

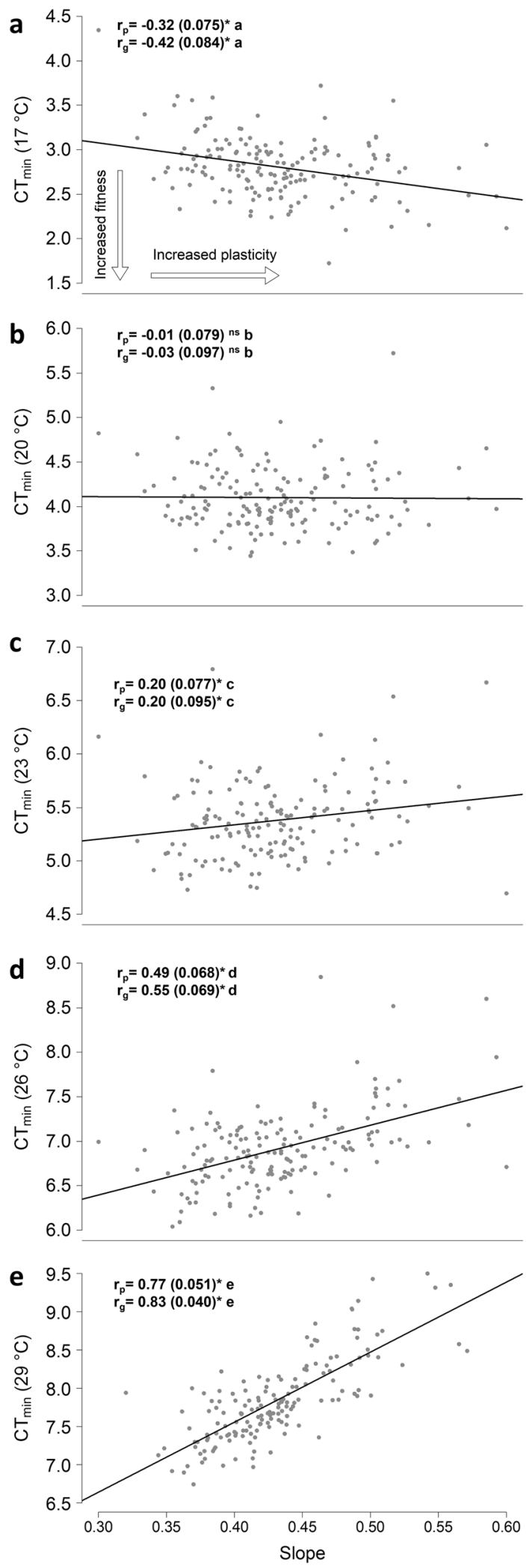

would likely have a fitness advantage when exposed to low temperatures, as they would remain the most cold tolerant (i.e., maintain relatively lower $\mathrm{CT}_{\min }$ at higher
Fig. 3 Correlations between $\mathrm{CT}_{\min }$ at the individual temperatures and slope of $\mathrm{CT}_{\min }$ across temperatures: a $17^{\circ} \mathrm{C}, \mathbf{b} 20^{\circ} \mathrm{C}, \mathbf{c} 23^{\circ} \mathrm{C}, \mathbf{d ~} 26^{\circ} \mathrm{C}$ and e $29^{\circ} \mathrm{C}$. Each dot represents one DGRP line, and the full black lines are the regression lines visualising the phenotypic correlations. In each environment Pearson's product moment correlation coefficients are shown for the phenotypic correlations, $r_{p}$ (SE in parenthesis) along with the printed genetic correlations, $r_{g}$ (SE in parenthesis) between $\mathrm{CT}_{\text {min }}$. Asterisks designate correlations that are significantly different from 0 , i.e., the estimate deviate more than $1.645 \times \mathrm{SE}$ from 0 $(p<0.05)$. Correlations that are not different from 0 are designated "ns". In pairwise comparisons of the correlations, significantly different correlations are denoted with separate letters after the correlation coefficients, e.g., $r_{g}\left(\mathrm{CT}_{\min } 17^{\circ} \mathrm{C}\right.$, slope $)$ vs. $r_{g}\left(\mathrm{CT}_{\min } 20^{\circ} \mathrm{C}\right.$, slope $)$. Phenotypic and genetic correlations are compared separately. Comparisons of these correlations are based on Fisher's $z$ transformation $(n=166)$. The two arrows in panel a shows the direction of increased fitness (here opposite of increased $\mathrm{CT}_{\min }$ ) and increased plasticity of $\mathrm{CT}_{\text {min }}$, respectively, for all panels

developmental temperatures). However, as indicated by the negative correlation between slope and $\mathrm{CT}_{\min }$ at $17{ }^{\circ} \mathrm{C}$, DGRP lines with low $\mathrm{CT}_{\min }$ reared at this temperature, typically had a high $\mathrm{CT}_{\text {min }}$ (low cold tolerance) when they developed at higher temperatures, indicative of a trade-off (Fig. 3a). At low developmental temperatures, lines with low $\mathrm{CT}_{\text {min }}$ and a steep slope could be considered "specialists"; i.e., they performed relatively better in one environment, but relatively poorly across environments. At higher developmental temperatures $\left(23,26\right.$ and $\left.29^{\circ} \mathrm{C}\right)$, we found a positive correlation between $\mathrm{CT}_{\min }$ and the plastic response, suggesting that high cold tolerance (low $\mathrm{CT}_{\min }$ ) at high developmental temperatures was associated with low plasticity (Fig. 3c-e). Thus, genotypes with low $\mathrm{CT}_{\min }$ at higher developmental temperatures could also be considered as the "specialists" because they performed relatively better in one environment. This shift in sign of the correlations reflects a reversal in the ordering of genotypes for cold resistance across the thermal gradient, with a tipping point at $20^{\circ} \mathrm{C}$, when there was no correlation between cold tolerance and plasticity.

These genotypic differences in constraints may reflect differential environmental sensitivity and suggests that the fitness effect of plasticity depends heavily on the environment where genotypes are reared. Thus, the fitness landscape of cold tolerance needs to be considered in the context of environmental frequencies, i.e., how likely are individuals reared under one set of thermal conditions to encounter subsequent extreme cold conditions (Ayrinhac et al. 2004)? Our findings support those of Levine et al. (2011) who suggested a "directionality" of plasticity in gene expression depending on the thermal environment commonly encountered in the native range of populations of D. melanogaster. This has implications for understanding developmental processes in variable environments (Stearns et al. 1991), and also for understanding local adaptation in thermal tolerances in populations with a broad geographic 
distribution along an environmental cline (Ayrinhac et al. 2004; Levine et al. 2011; Cooper et al. 2012; Kellermann et al. 2012; Kristensen et al. 2015). Our results indicate that the quantitative genetic basis of basal cold tolerance and plastic cold tolerance is highly environment specific, which is important for predicting selection responses in natural and variable environments (Sgrò and Hoffmann 2004; Agrawal and Stinchcombe 2009).

Most studies investigating phenotypic and genetic correlations and/or candidate genes between thermal environments focus on only two temperatures (Guerra et al. 1997; Ellers and Driessen 2011; Ketola et al. 2012) or investigate two settings such as field vs. lab conditions (Kristensen et al. 2008; Overgaard et al. 2010), and thus do not provide a comprehensive picture of changing genetic correlations and architectures across environments. A meta-analysis by Charmantier and Garant (2005) specifically called for approaches that consider genetic variability under multiple conditions, and ideally along a continuous environmental gradient such as in the present study. Because correlations within and between traits can change between environments or even shift in sign (Sgrò and Hoffmann 2004; Agrawal and Stinchcombe 2009) as evident in this study, we reiterate this call and caution about genetic constraints and trade-offs deduced from studies that consider only one or two environments.

In conclusion, we showed that the expression of additive genetic variation for cold tolerance was environment dependent, and that evolvability of cold resistance decreased with increasing developmental temperatures. We also showed that the relationship between basal and plastic cold tolerance was affected by the environment, and even switched sign across a gradient of developmental temperatures. We further provided evidence for an evolutionary trade-off between cold tolerance in one environment and cold tolerance across environment. It will be interesting to test for these patterns in relevant ecological settings, such as in populations exposed to strong diurnal and seasonal temperature fluctuations.

Acknowledgements DGRP stocks were obtained from the Bloomington Drosophila Stock Centre (NIH P40OD018537). We thank Iben R. Jensen, Helle Blendstrup, Susan M. Hansen, and Iben V. Nielsen for laboratory assistance. This research was financed by the Danish Natural Science Research Council through a Sapere aude stipend to TNK (DFF-4002-00036), the Science Industry Endowment Fund and a grant from the Velux Visiting Professor programme to $\mathrm{AAH}$, and by the Danish Strategic Research Council (GenSAP: Centre for Genomic Selection in Animals and Plants, contract 12-132452) to PS.

\section{Compliance with ethical standards}

Conflict of interest The authors declare that they have no conflict of interest.

\section{References}

Agrawal AF, Stinchcombe JR (2009) How much do genetic covariances alter the rate of adaptation? Proc R Soc B Biol Sci 276:1183-1191

Araújo MB, Ferri-Yáñez F, Bozinovic F, Marquet PA, Valladares F, Chown SL (2013) Heat freezes niche evolution. Ecol Lett 16:1206-1219

Auld JR, Agrawal AA, Relyea RA (2010) Re-evaluating the costs and limits of adaptive phenotypic plasticity. Proc R Soc B Biol Sci 277:503-511

Ayrinhac A, Debat V, Gibert P, Kister A-G, Legout H, Moreteau B et al. (2004) Cold adaptation in geographical populations of Drosophila melanogaster: phenotypic plasticity is more important than genetic variability. Funct Ecol 18:700-706

Bastide H, Lange JD, Lack JB, Yassin A, Pool JE (2016) A variable genetic architecture of melanic evolution in Drosophila melanogaster. Genetics 204:1307-1319

Bay RA, Rose N, Barrett R, Bernatchez L, Ghalambor CK, Lasky JR et al. (2017) Predicting responses to contemporary environmental change using evolutionary response architectures. Am Nat 189:463-473

Bijlsma R, Loeschcke V (2005) Environmental stress, adaptation and evolution: an overview. J Evol Biol 18:744-9

Bourret A, Garant D (2015) candidate gene-environment interactions and their relationships with timing of breeding in a wild bird population Ecol Evol 5:3628-3641

Bubliy OA, Kristensen TN, Kellermann V, Loeschcke V (2012) Humidity affects genetic architecture of heat resistance in Drosophila melanogaster. J Evol Biol 25:1180-1188

Calosi P, Bilton DT, Spicer JI (2008) Thermal tolerance, acclimatory capacity and vulnerability to global climate change. Biol Lett 4:99-102

Charmantier A, Garant D (2005) Environmental quality and evolutionary potential: lessons from wild populations. Proc R Soc B Biol Sci 272:1415-1425

Chown SL, Hoffmann AA, Kristensen TN, Angilletta MJ, Stenseth N, Pertoldi C (2010) Adapting to climate change: a perspective from evolutionary physiology. Clim Res 43:3-15

Comte L, Olden JD (2017) Evolutionary and environmental determinants of freshwater fish thermal tolerance and plasticity. Glob Change Biol 23:728-736

Cooper BS, Tharp JM, Jernberg II, Angilletta MJ (2012) Developmental plasticity of thermal tolerances in temperate and subtropical populations of Drosophila melanogaster. J Therm Biol 37:211-216

Czesak ME, Fox CW, Wolf JB (2006) Experimental evolution of phenotypic plasticity: how predictive are cross-environment genetic correlations? Am Nat 168:323-35

David JR, Gibert P, Gravot E, Petavy G, Morin JP, Karan D et al. (1997) Phenotypic plasticity and developmental temperature in drosophila: analysis and significance of reaction norms of morphometrical traits. J Therm Biol 22:441-451

Dingemanse NJ, Van der Plas F, Wright J, Reale D, Schrama M, Roff DA et al. (2009) Individual experience and evolutionary history of predation affect expression of heritable variation in fish personality and morphology. Proc R Soc B Biol Sci 276:1285-1293

Ellers J, Driessen G (2011) Genetic correlation between temperatureinduced plasticity of life-history traits in a soil arthropod. Evol Ecol 25:473-484

Etterson JR, Shaw RG (2001) Constraint to adaptive evolution in response to global warming. Science 294:151-154

Falconer DS, Mackay TFC (1996) Introduction to quantitative genetics, 4th edn. Longman Publishing Group, Harlow, UK 
Feldman MJ, Paul RE, Banan D, Barrett JF, Sebastian J, Yee MC et al. (2017) Time dependent genetic analysis links field and controlled environment phenotypes in the model $\mathrm{C} 4$ grass Setaria. PLOS Genet 13:e1006841

Franke K, Dierks A, Fischer K (2012) Directional selection on cold tolerance does not constrain plastic capacity in a butterfly. BMC Evol Biol 12:235

Gebhardt-Henrich SG, van Noordwijk AJ (1991) Nestling growth in the Great Tit I. Heritability estimates under different environmental conditions. J Evol Biol 4:341-362

Gerken AR, Eller OC, Hahn DA, Morgan TJ (2015) Constraints, independence, and evolution of thermal plasticity: probing genetic architecture of long- and short-term thermal acclimation. Proc Natl Acad Sci USA 112:4399-4404

Goenaga J, Mensch J, Fanara JJ, Hasson E (2012) The effect of mating on starvation resistance in natural populations of Drosophila melanogaster. Evol Ecol 26:813-823

Guerra D, Cavicchi S, Krebs R, Loeschcke V (1997) Resistance to heat and cold stress in Drosophila melanogaster: intra and inter population variation in relation to climate. Genet Sel Evol 29:497-510

Gunderson AR, Stillman JH (2015) Plasticity in thermal tolerance has limited potential to buffer ectotherms from global warming. Proc R Soc B Biol Sci 282:1-8

van Heerwaarden B, Sgrò CM (2011) The effect of developmental temperature on the genetic architecture underlying size and thermal clines in Drosophila melanogaster and D. simulans from the east coast of Australia. Evolution 65:1048-1067

Hercus MJ, Hoffmann AA (2000) Maternaland grandmaternal age influence offspring fitness in Drosophila. Proc R Soc B Biol Sci 267:2105-2110

Hoffmann AA (2010) Physiological climatic limits in Drosophila: patterns and implications. J Exp Biol 213:870-880

Hoffmann AA, Merilä J (1999) Heritable variation and evolution under favourable and unfavourable conditions. Trends Ecol Evol 14:96-101

Hoffmann AA, Merilä J, Kristensen TN (2016) Heritability and evolvability of fitness and nonfitness traits: lessons from livestock. Evolution 70:1770-1779

Hoffmann AA, Parsons PA (1991) Evolutionary genetics and environmental stress. Oxford University Press, Oxford, UK

Hoffmann AA, Sørensen JG, Loeschcke V (2003) Adaptation of Drosophila to temperature extremes: bringing together quantitative and molecular approaches. J Therm Biol 28:175-216

Houle D (1992) Comparing evolvability and variability of quantitative traits. Genetics 130:195-204

Huang W, Massouras A, Inoue Y, Peiffer J, Ràmia M, Tarone AM et al. (2014) Natural variation in genome architecture among 205 Drosophila melanogaster Genetic Reference Panel lines. Genome Res 24:1193-1208

Husby A, Visser ME, Kruuk LEB (2011) Speeding up microevolution: the effects of increasing temperature on selection and genetic variance in a wild bird population. PLOS Biol 9:e1000585

de Jong G (1990) Quantitative genetics of reaction norms. J Evol Biol $3: 447-468$

Kadarmideen HN, Von Rohr P, Janss LLG (2006) From genetical genomics to systems genetics: potential applications in quantitative genomics and animal breeding. Mamm Genome 17:548-564

Kellermann V, Overgaard J, Hoffmann AA, Fløjgaard C, Svenning JC, Loeschcke V (2012) Upper thermal limits of Drosophila are linked to species distributions and strongly constrained phylogenetically. Proc Natl Acad Sci USA 109:16228-33

Kellett M, Hoffmann AA, Mckechnie SW (2005) Hardening capacity in the Drosophila melanogaster species group is constrained by basal thermotolerance. Funct Ecol 19:853-858
Ketola T, Kellermann V, Kristensen TN, Loeschcke V (2012) Constant, cycling, hot and cold thermal environments: strong effects on mean viability but not on genetic estimates. J Evol Biol 25:1209-1215

Kimura MT (2004) Cold and heat tolerance of drosophilid flies with reference to their latitudinal distributions. Oecologia 140:442-449

Kristensen TN, Hoffmann AA, Overgaard J, Sørensen JG, Hallas R, Loeschcke V (2008) Costs and benefits of cold acclimation in field-released Drosophila. Proc Natl Acad Sci USA 105:216-221

Kristensen TN, Overgaard J, Lassen J, Hoffmann AA, Sgrò C (2015) Low evolutionary potential for egg-to-adult viability in Drosophila melanogaster at high temperatures. Evolution 69:803-814

Levine MT, Eckert ML, Begun DJ (2011) Whole-genome expression plasticity across tropical and temperate Drosophila melanogaster populations from eastern Australia. Mol Biol Evol 28:249-256

Mackay TFC, Richards S, Stone EA, Barbadilla A, Ayroles JF, Zhu D et al. (2012) The Drosophila melanogaster Genetic Reference Panel. Nature 482:173-178

Madsen P, Jensen J, Thompson R (1994). Estimation of (co)-variance components by REML in multivariate mixed linear models using average of observed and expected information. In: FifthWorld Congress of Genetics Applied to Livestock Production, Guelph, Ontario, Canada, pp 455-462.

Manenti T, Sørensen JG, Moghadam NN, Loeschcke V (2016) Few genetic and environmental correlations between life-history and stress resistance traits affect adaptation to fluctuating thermal regimes. Heredity 117:149-154

Murren CJ, Auld JR, Callahan H, Ghalambor CK, Handelsman CA, Heskel MA et al. (2015) Constraints on the evolution of phenotypic plasticity: limits and costs of phenotype and plasticity. Heredity 115:293-301

Møller A, Swaddle K (1998) Asymmetry, developmental stability and evolution. Oxford University Press, Oxford, UK

Noh S, Everman ER, Berger CM, Morgan TJ (2017) Seasonal variation in basal and plastic cold tolerance: adaptation is influenced by both long- and short-term phenotypic plasticity. Ecol Evol 7:5248-5257

Nyamukondiwa C, Terblanche JS, Marshall KE, Sinclair BJ (2011) Basal cold but not heat tolerance constrains plasticity among Drosophila species (Diptera: Drosophilidae). J Evol Biol 24:1927-1938

Overgaard J, Kristensen TN, Mitchell KA, Hoffmann AA (2011) Thermal tolerance in widespread and tropical Drosophila species: does phenotypic plasticity increase with latitude? Am Nat 178:80-96

Overgaard J, Kristensen TN, Sørensen JG (2012) Validity of thermal ramping assays used to assess thermal tolerance in arthropods. PLOS ONE 7:1-7

Overgaard J, Sørensen JG, Jensen LT, Loeschcke V, Kristensen TN (2010) Field tests reveal genetic variation for performance at low temperatures in Drosophila melanogaster. Funct Ecol 24:186-195

R Core Team (2017) R: a language and environment for statistical computing. Version 3.4.0. R Foundation for Statistical Computing, Vienna, Austria, http://www.r-project.org/

Rellstab C, Fischer MC, Zoller S, Graf R, Tedder A, Shimizu KK et al. (2017) Local adaptation (mostly) remains local: reassessing environmental associations of climate-related candidate SNPs in Arabidopsis halleri. Heredity 118:193-201

Roff DA (2002) Life history evolution. Sinauer Associates, Sunderland, UK

Rohde PD, Gaertner B, Wards K, Sørensen P, Mackay TFC (2017) Genomic analysis of genotype-by-social environment interaction for Drosophila melanogaster aggressive behavior. Genetics 206:1969-1984 
Schou MF, Kristensen TN, Kellermann V, Schlötterer C, Loeschcke V (2014) A Drosophila laboratory evolution experiment points to low evolutionary potential under increased temperatures likely to be experienced in the future. J Evol Biol 27:1859-1868

Schou MF, Mouridsen MB, Sørensen JG, Loeschcke V (2017) Linear reaction norms of thermal limits in Drosophila: predictable plasticity in cold but not in heat tolerance. Funct Ecol 31:934-945

Sgrò CM, Blows MW (2004) The genetic covariance among clinal environments after adaptation to an environmental gradient in Drosophila serrata. Genetics 167:1281-1291

Sgrò CM, Hoffmann AA (2004) Genetic correlations, trade-offs and environmental variation. Heredity 93:241-248

Sheldon BC, Kruuk LEB, Merilä J (2003) Natural selection and inheritance of breeding time and clutch size in the collared flycatcher. Evolution 57:406-420

Spichtig M, Kawecki TJ (2004) The maintenance (or not) of polygenic variation by soft selection in heterogeneous environments. Am Nat 164:70-84

Stearns S, de Jong G, Newman B (1991) The effects of phenotypic plasticity on genetic correlations. Trends Ecol Evol 6: $122-126$

Steinger T, Roy BA, Stanton ML (2003) Evolution in stressful environments II: adaptive value and costs of plasticity in response to low light in Sinapis arvensis. J Evol Biol 16:313-323

Stillman JH (2003) Acclimation capacity underlies susceptibility to climate change. Science 301:65

Stinchcombe JR, Izem R, Heschel MS, McGoey BV, Schmitt J (2010) Across environment genetic correlations and the frequency of selective environments shape the evolutionary dynamics of growth rate in impatiens capensis. Evolution 64:2887-2903

Strachan LA, Tarnowski-Garner HE, Marshall KE, Sinclair BJ (2011) The evolution of cold tolerance in Drosophila larvae. Physiol Biochem Zool 84:43-53

Sunday JM, Bates AE, Dulvy NK (2011) Global analysis of thermal tolerance and latitude in ectotherms. Proc R Soc B Biol Sci 278:1823-1830

Swindell WR, Bouzat JL (2006) Associations between environmental stress, selection history, and quantitative genetic variation in Drosophila melanogaster. Genetica 127:311-20

Talloen W, Van Dongen S, Van Dyck H, Lens L (2009) Environmental stress and quantitative genetic variation in butterfly wing characteristics. Evol Ecol 23:473-485

Telonis-Scott M, Hallas R, McKechnie SW, Wee CW, Hoffmann AA (2009) Selection for cold resistance alters gene transcript levels in Drosophila melanogaster. J Insect Physiol 55:549-555
Tonsor SJ, Elnaccash TW, Scheiner SM (2013) Developmental instability is genetically correlated with phenotypi plasticity, constraining heritability, and fitness. Evolution 67:2923-2935

Valladares F, Sanchez-Gomez D, Zavala MA (2006) Quantitative estimation of phenotypic plasticity: bridging the gap between the evolutionary concept and its ecological applications. J Ecol 94:1103-1116

VanRaden PM (2008) Efficient methods to compute genomic predictions. J Dairy Sci 91:4414-4423

Via S, Lande R (1985) Genotype-environment interaction and the evolution of phenotypic plasticity. Evolution 39:505-522

Vieira C, Pasyukova EG, Zeng ZB, Hackett JB, Lyman RF, Mackay TF (2000) Genotype-environment interaction for quantitative trait loci affecting life span in Drosophila melanogaster. Genetics 154:213-227

Visscher PM, Hill WG, Wray NR (2008) Heritability in the genomics era-concepts and misconceptions. Nat Rev Genet 9:255-266

Williams CM, Henry HAL, Sinclair BJ (2014) Cold truths: how winter drives responses of terrestrial organisms to climate change. Biol Rev 90:214-235

Wilson AJ, Pemberton JM, Pilkington JG, Coltman DW, Mifsud DV, Clutton-Brock TH et al. (2006) Environmental coupling of selection and heritability limits evolution. PLoS Biol 4:1270-1275

Wood CW, Brodie ED (2016) Evolutionary response when selection and genetic variation covary across environments. Ecol Lett 19:1189-1200

Zhao K, Tung C-W, Eizenga GC, Wright MH, Ali ML, Price AH et al. (2011) Genome-wide association mapping reveals a rich genetic architecture of complex traits in Oryza sativa. Nat Commun 2:467

Zhao W, Wang X, Wang H, Tian J, Li B, Chen L et al. (2016) Genome-wide identification of QTL for seed yield and yieldrelated traits and construction of a high-density consensus map for QTL comparison in Brassica napus. Front Plant Sci 7:17

Ørsted M, Malmendal A, Muñoz J, Kristensen TN (2018a) Metabolic and functional phenotypic profiling of Drosophila melanogaster reveals reduced sex differentiation under stressful environmental conditions. Biol J Linn Soc 123:155-162

Ørsted M, Rohde PD, Hoffmann AA, Sørensen P, Kristensen TN (2018b) Environmental variation partitioned into separate heritable components. Evolution 72:136-152

Ørsted M, Schou MF, Kristensen TN (2017) Biotic and abiotic factors investigated in two Drosophila species-evidence of both negative and positive effects of interactions on performance. Sci Rep 7:40132 